\title{
A CLASSIFICATION OF THE COMMUTATOR SUBGROUP OF THE GROUP OF A BOUNDARY LINK
}

\author{
BAI CHING CHANG
}

(Received 15 June 1972)

Communicated by G. E. Wall

In Neuwirth's book "Knot Groups" ([2]), the structure of the commutator subgroup of a knot is studied and characterized. Later Brown and Crowell refined Neuwith's result ([1], and we thus know that if $G$ is the group of a knot $K$, then $[G, G]$ is either free of rank $2 g$, where $g$ is the genus of $K$, or a nontrivial free product with amalgamation on a free group of rank $2 g$, and may be written in the form $\cdots * A * A * A * A * A * \cdots$, where $F$ is free of rank $2 g$, and the amalgamations are all proper and identical.

Neuwirth's method is quite elementary, and it seems easy to apply to the study of the commutator subgroup of a boundary link, as defined by Ralph H. Fox. With the help of the results of Brown and Crowell, we do get a characterization of the commutator subgroup of the group of a boundary link, as shown in the rest of the paper.

1. Construction of the covering space correspond to $[G, G]$

By a boundary link of two components we mean a link which is the boundary of two disjoint, orientable surfaces. We can assume these to be so chosen that if we keep one of them fixed, we cannot replace the other one by any surface with lower genus. To do this, it is sufficient to choose surfaces for which the sum of the genera is a minimum.

We now construct the covering space of a boundary link $L$ (of two components) corresponding to the commutator subgroup of $\pi\left(S^{1}-L\right)$. Let $S_{1}$ and $S_{2}$ be two orientable surfaces which span $L$. Take a countable number of disjoint copies of $X=\left(S^{3}\right.$ split along $\left.S_{1}, S_{2}\right)$. This means $X$ has on its boundary two copies of $S_{1}$ which we will denote by $S_{11}, S_{12}$, and two copies of $S_{2}$ which we will denote by $S_{21}, S_{22}$. Note that $S_{11} \cup S_{12} \cup S_{21} \cup S_{22}=\partial X$ and $\left(S_{11} \cup S_{21}\right) \cap$ $\left(S_{12} \cup S_{22}\right)=L$. Index the copies of $X$ by $(i, j)$, where $i, j$ are integers; we paste copy $X_{j}^{i}$ to copy $X_{j}^{i+1}$ along the "right hand" copy ${ }_{j}^{i} S_{11}$ of $S_{1}$ in $X_{j}^{i}$, and the 
"left hand" copy ${ }_{j}^{i+1} S_{12}$ of $S_{1}$ in $X_{j}^{i+1}$. We also paste copy $X_{j}^{i}$ to copy $X_{j+1}^{i}$ along right hand copy ${ }_{j}^{i} S_{21}$ of $S_{2}$ in $X_{j}^{i}$ to the left hand copy ${ }_{j+1}^{i} S_{22}$ of $S_{2}$ in $X_{j+1}^{i}$. The common copy of $L$ in each $X_{j}^{i}$ is then removed.

$$
\begin{array}{c|cc}
X_{j-1}^{i} \quad{ }_{j-1}{ }^{i} S_{21} \mid{ }_{j}^{i} S_{22} & \frac{{ }_{j}^{i+1} S_{j 1}^{i+1} S_{12}}{X_{j}^{i}} \\
\frac{{ }_{j}^{i} S_{12}}{{ }^{i-1} S_{j 1}} \\
X_{j}^{i-1} S_{j 1}^{i} S_{21}
\end{array}
$$

The resulting space $\tilde{M}$ is the covering space of $X$ that corresponds to the commutator subgroup of $\pi\left(S^{3}-L\right)$.

LEMMA 1. The inclusion map $h: S_{11} \rightarrow S_{11} \cup S_{12}$ induces a monomorphism $h^{*}: \pi\left(S_{11}\right) \rightarrow \pi\left(S_{11} \cup S_{12}\right)$.

Proof. If $S_{11}$ is the genus greater than zero, then using the van Kampen theorem, $\pi\left(S_{11} \cup S_{12}\right)=\pi\left(S_{11}\right)_{\pi\left(S_{11} \cap S_{12}\right)}^{*} \pi\left(S_{12}\right)$, so $h^{*}$ is a monomorphism.

If $S_{11}$ is of genus zero, then $\pi\left(S_{11}\right)=0, h^{*}$ is automatically a monomorphism.

LEMMA 2. The inclusion map $v: S_{11} \rightarrow X$ induces a monomorphism $v^{*}$, $\pi\left(S_{11}\right) \rightarrow \pi(X)$.

Proof. Suppose that the lemma is false, and that $\alpha$ is a closed curve on $S_{11}$ such that $\alpha \simeq 0$ in $X, \alpha \neq 0$ on $S_{11}$. According to lemma $1, \alpha \neq 0$ on $S_{11} \cup S_{12}$. Since $S_{11}$ is polyhedral, $\alpha$ may be assumed polyhedral so that $X$ and $\alpha$ satisfy the hypothesis of the loop theorem. ([3], Theorem 15.1 and Theorem 1), thus we may assume that $\alpha$ is simple. According to Dehn's lemma, $\alpha$ bounds a nonsingular polyhedral disc in $X$. Since $S_{11} \cup S_{12}$ is also polyhedral, we may assume that this disc does not intersect $S_{11} \cup S_{12}$ except in $\alpha$. If we cut $S_{11}$ along $\alpha$, and sew discs to both sides of the cut we obtain a new surface $S_{11}^{\prime}$ which has the same boundary as $S_{11}$, and is disjoint from $S_{2}$. If the curve $\alpha$ separates $S_{11}$, then because $\alpha \neq 0$ on $S_{11}$, the new surface $S_{11}^{\prime}$ has lower genus than $S_{1}$, which contradicts the assumption on $S_{1}$. If $\alpha$ does not separate $S_{1}$, then compare the Euler characteristic $\chi\left(S_{11}\right)$ of $S_{11}$ with $\chi\left(S_{11}^{\prime}\right)$, the Euler characteristic of $S_{11}^{\prime}$. Since the cut adds one vertex, one edge, and two faces, $\chi\left(S_{11}^{\prime}\right)-\chi\left(S_{11}\right)=2$ hence $S_{11}^{\prime}$ has lower genus than $S_{11}$, again a contradiction, so the lemma is proved.

Lemmas 1 and 2 obviously remain valid if $S_{12}, S_{21}, S_{22}$ are substituted for $S_{11}$. Lemma 2 also remains valid if $L$ is removed from $X$. 
We will now calculate the commutator subgroup of $\pi\left(S^{3}-L\right)$. Before we do that, we need some algebraic preliminaries.

Let $A$ be an arbitrary group, $C, D, E, F$ subgroups of $A, \phi: C \rightarrow D, \psi: E \rightarrow F$ both isomorphisms. Take a countable number of copies of them, index by $(i, j)$, where $i, j$ are integers, $C_{i j}, D_{i j}, E_{i j}, F_{i j}$ are now considered as subgroups of $A_{i j}$, but $\phi_{i j}\left(\psi_{i j}\right)$ is considered as an isomorphism of $C_{i j}$ with $D_{i j+1}\left(E_{i j}\right.$ with $\left.F_{i+1, j}\right)$. We now define $\bar{A}$ as the group generated by all $A_{i j}$, subject to the relation $c\left(\phi_{i j}(c)\right)^{-1}=1$ for $c \in C_{i j}, e\left(\psi_{i j}(e)\right)^{-1}=1$ for $e \in E_{i j}$.

For each $i$, let $\bar{A}_{i}$ be the group generated by $A_{i j}(-\infty<j<\infty)$, subject to the relation $c\left(\phi_{i j}(c)\right)^{-1}=1, c \in C_{i j}$ (thus $A$ is a free product with amalgamation) We have an obvious map: $\bar{A}_{i} \rightarrow \bar{A}$, we shall use $\overline{\bar{A}}_{i}$ to denote the image of $\bar{A}_{i}$ in $\bar{A}$. Clearly $\overline{\bar{A}}_{i}$ 's are all (canonically) isomorphic.

Let $\bar{E}_{i}, F_{i}$ be the subgroup of $\bar{A}_{i}$ which is generated by $E_{i j}, F_{i j}(-\infty<j<\infty)$. $\overline{\bar{E}}_{i}, \overline{\bar{F}}_{i} \subset \overline{\bar{A}}_{i}$ is the image of $\bar{E}_{i}, F_{i}$ in $\overline{\bar{A}}_{i}$. We define a map $\overline{\bar{\psi}}_{i}: \overline{\bar{E}}_{i} \rightarrow \overline{\bar{F}}_{i+1}$ by $\bar{\psi}_{i}([c])=\left[\psi_{i j}(c)\right]$, where $c \in E_{i j}$. We need to check whether this map is well defined. Suppose $\left[c_{1} c_{2} \cdots c_{n}\right]=\left[d_{1} d_{2} \cdots d_{m}\right]$ in $\overline{\bar{E}}_{i}$, this means $\left[c_{1} c_{2} \cdots c_{n}\right]=$ $\left[d_{1} d_{2} \cdots d_{m}\right]$ in $\bar{A}$. From the definition of $\bar{A}$, we know that $\left[c_{1} c_{2} \cdots c_{n}\right]=$ $\left[\psi_{i \beta_{1}}\left(c_{1}\right) \psi_{i \beta_{2}}\left(c_{2}\right) \cdots \psi_{i \beta_{n}}\left(c_{n}\right)\right]$ and $\left[d_{1} d_{2} \cdots d_{m}\right]=\left[\psi_{i t_{1}}\left(d_{1}\right) \psi_{i t_{2}}\left(d_{2}\right) \cdots \psi_{i t_{m}}\left(d_{m}\right)\right.$ if $c_{l} \in E_{i \beta_{l}}, d_{k} \in E_{i t_{k}}$. So $\left[\psi_{i \beta_{1}}\left(c_{1}\right) \psi_{i \beta_{2}}\left(c_{2}\right) \cdots \psi_{i \beta_{n}}\left(c_{n}\right)\right]=\left[\psi_{i t_{1}}\left(d_{1}\right) \psi_{i t_{2}}\left(d_{2}\right) \cdots \psi_{i t_{m}}\left(d_{m}\right)\right]$, so our map is well defined.

It is clear that $\overline{\bar{\psi}}_{i}$ 's are isomorphisms. In fact $\psi_{i j}^{-1}: \overline{\bar{E}}_{i} \rightarrow \bar{F}_{i}$ is defined by simply mapping $[d], \mathrm{d} \in F_{i+1, j}$, to $\left[\psi_{i j}^{-1}(d)\right]$.

Let $\tilde{A}$ be the group generated by all $\overline{\bar{A}}_{i}(-\infty<i<\infty)$, under the relation $c\left(\overline{\bar{\psi}}_{i}(c)\right)^{-1}=1$ for $c \in \bar{E}_{i}$. Clearly we have a map from $\tilde{A}$ to $\bar{A}$ which is onto. We also have a natural map from $A_{i j}$ to $\tilde{A}$ which can easily be extended to a map from $\bar{A}$ to $\tilde{A}$. We thus see that $\tilde{A}=\bar{A}$.

We now go back to the calculation of the commutator subgroups of $\pi\left(S^{3}-L\right)$.

Let $A=\pi(X-L), C=$ the image of $\pi\left(S_{21}-L\right)$ in $A, D=$ the image of $\pi\left(S_{22}-L\right)$ in $A, E=$ the image of $\pi\left(S_{11}-L\right)$ in $A, F=$ the image of $\pi\left(S_{12}-L\right)$ in $A, \phi, \psi$ defined in the natural way.

With these notations, using van Kampen's original version of van Kampen theorem and the algebraic results we just mentioned, it is clear that $\pi(\tilde{M})=\bar{A} * B$, where $B$ is the free group with a countable number of generators.

Lemma 3. The map $\bar{A}_{i} \rightarrow \bar{A}$ is injective.

Proof. Let $\mathscr{A}$ be a loop in $\bigcup_{j=-\infty}^{\infty} X_{i j}-L$ which represents the trivial element in $\bar{A}$. We can represent $\mathscr{A}$ by a simple polygonal loop in $\bigcup_{j=-\infty}^{\infty} X_{i j}-L$. By using Dehn's lemma, it bounds a nonsingular disc $\mathscr{D}$ in $\tilde{M}$. We can even assume that $\mathscr{D}$ and ${ }_{q}^{p} S_{r s}-L(-\infty<p, q<\infty ; r, s=1,2)$ intersect in general position, so their intersection is a finite number of simple loops $l_{1}, l_{2}, \cdots, l_{r}$. We shall prove that $\mathscr{A}$ is trivial in $\bar{A}_{i}$ by using induction on $r$. 
When $r=1, l_{1}$ lies on ${ }^{i} S_{11}-L$ (or ${ }_{j}^{i} S_{21}-L$ ) for some $-\infty<j<\infty$. Since the $\operatorname{map} \pi\left({ }_{q}^{p} S_{s t}-L\right) \rightarrow \pi\left(X_{q^{\prime}}^{p^{\prime}}-L\right)\left(p=p^{\prime}, q^{\prime}=q\right.$ or $q+1$ if $s=2, p^{\prime}=p$ or $p+1, q^{\prime}=q$ if $s=1$ ) is injective, $l_{1}$ bounds a disc on ${ }_{j}^{i} S_{11}-L$ (or ${ }_{j}^{i} S_{21}-L$ ). So we can replace $\mathscr{D}$ by a new disc $\mathscr{D}^{\prime}$ which does not intersect ${ }_{j}^{i} S_{11}-L$ or ${ }_{j}^{i} S_{21}-L$. This imples that $\mathscr{A}$ represtnts the trivial element in $\bar{A}_{i}$.

Now suppose that we have proved that $\mathscr{A}$ is trivial in $\bar{A}_{i}$ when $r \leqq k$. For $r=k+1$, let $l_{1}$ be a loop which is innermost in the sense that it bounds no other $l_{t}, 1<t \leqq k+1$, on the surface ${ }_{q}^{p} S_{11}-L$ or ${ }_{q}^{p} S_{21}-L$ wherever it lies. Since $l_{1}$ still bounds a disc $\mathscr{D}_{1}$ in $\tilde{M}$ and since $\mathscr{D}_{1}$ intersect ${ }_{n}^{m} S_{11}-L$ and ${ }_{n}^{m} S_{12}-L$ for $-\infty<m, n<\infty$ in at most $k$ loops, $l_{1}$ will bound a disc in ${ }_{q}^{p} S_{11}-L$ or ${ }_{q}^{p} S_{21}-L$ (we are using the fact that map $\pi\left({ }_{q}^{p} S_{s 1}-L\right) \rightarrow \pi\left(X_{q^{\prime}}^{p^{\prime}}-L\right)$ is injective). This implies that we can replace $D$ by a disc $D^{\prime}$ which intersects ${ }_{n}^{m} S_{11}-L$, ${ }_{n}^{m} S_{21}-L$ for $-\infty<m, n<\infty$ in at most $k$ simple loops, thus by our induction hypothesis, $\mathscr{A}$ represents the trivial element in $\bar{A}_{i}$.

Clearly $\bar{A}_{i}$ is itself a free product with amalgamation (which may be improper). And $\bar{A}=\tilde{A}$ is also a free product with amalgamation.

$$
\begin{aligned}
& \bar{A}_{i}=\cdots A_{C_{i-2}}^{A_{D_{i-1}}}{ }^{A_{i-1} C_{i-1}}{ }^{*} \underset{D_{i 0}}{A_{i 0} C_{C_{20}}} * \underset{D_{i 1}{ }^{A_{11}} C_{C_{i 1}}}{*}{ }_{D_{i 2}}^{A_{i 2}} \cdots \\
& \bar{A}=\cdots \bar{A}_{-2} \overline{\bar{E}}_{-2}{ }^{*} \overline{\bar{F}}_{-1} \bar{A}_{-1} \overline{\bar{E}}_{-1}{ }^{*} \overline{\bar{F}}_{0} \bar{A}_{0} \overline{\bar{E}}_{0}{ }^{*} \overline{\bar{F}}_{1} \bar{A}_{1} \overline{\bar{E}}_{1}{ }^{*}{ }_{\bar{F}_{2}} \bar{A}_{2} \cdots
\end{aligned}
$$

Lemma 4. Let $B$ be a compact, connected 3-manifold, and let $S$ be a nonvoid compact connected 2-manifold properly contained in $\partial B$ such that

(i) $\chi(S) \geqq \chi(B)$

(ii) $\pi(S) \rightarrow \pi(B)$ is onto

then $S$ and $T=\mathrm{CL}(\partial B-S)$ are strong deformation retracts of $B$.

In the following diagram, $G_{i}, H_{i}$ are groups, $g_{i}, h_{i}, f_{i}$ are homomorphism, and form a commutative diagram; $f_{*}$ is the induced homomorphism from the direct limit of the top row to the direct limit of the bottom row:

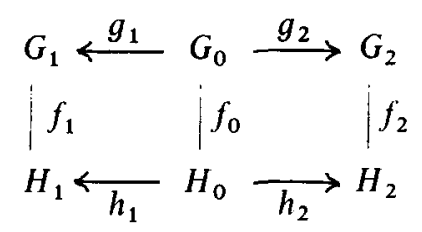

LEMMA 5. If $h_{1}$ and $h_{2}$ are one-to-one and if $f_{0}$ and $f_{*}$ are onto, then $f_{1}$ and $f_{2}$ are onto.

These two lemmas are Theorem $(1.1)$ and proposition $(2,4)$ of Brown and Crowell's paper.

We now state the main result of this paper: 
THEOREM. The commutator subgroup of $\pi\left(S^{3}-L\right)$ can be written in the form $\bar{A} * B$, where $B$ is the free group with a countable number of generators. If one of $S_{1}$ and $S_{2}$ is a disc, then $\bar{A}$ may be written in the form

$$
\bar{A}=\cdots * \overline{\bar{A}} * \overline{\bar{A}} * \overline{\bar{A}} * \overline{\bar{A}} * \overline{\bar{A}} * \cdots
$$

where $\overline{\bar{A}}$ is either free of even rank, or a nontrivial free product with amalgamation on free groups of even rank, and may be written in the form:

$$
\overline{\bar{A}}=\cdots \underset{F}{*} A \underset{F}{A} A \underset{F}{A} \underset{F}{A *} * \cdots
$$

where $F$ is free of even rank, and the amalgamations are all proper and identical. If none of $S_{1}$ and $S_{2}$ is a disc, then $\bar{A}$ is a nontrivial free product with amalgamation, and may be written in the form

$$
\bar{A}=\cdots * \overline{\bar{A}}_{\overline{\bar{E}}} * \overline{\overline{\bar{F}}}_{\overline{\bar{E}}} * \overline{\overline{\bar{F}}}_{\overline{\bar{E}}} * \overline{\overline{\bar{F}}}_{\overline{\overline{\mathrm{A}}}} *{ }_{\overline{\overline{\mathrm{F}}}} \overline{\bar{A}} * \cdots
$$

where $\overline{\bar{A}}$ itself is a nontrivial free product with amalgamation on free groups of even rank, and may be written in the form

$$
\overline{\bar{A}}=\cdots * A \underset{C}{A} A_{C} * A C_{D} * A * A * \cdots
$$

where $C$ or $D$ are free of even rank, the amalgamations are all proper and identical.

Proof. The case when one of $S_{1}$ and $S_{2}$ is a disc is rather trivial to see. If none of $S_{1}$ and $S_{2}$ is a disc, since $\chi\left(S^{3}-L\right)=\chi\left(S_{1}\right)+\chi\left(S_{2}\right)$, Lemma 4 shows that if $\pi\left(S_{i j}\right) \rightarrow \pi\left(X_{q}^{p}\right)$ is onto, $\partial X_{q}^{p}-S_{i j}$ has to be connected, which is not true. This proves $\overline{\bar{A}}$ has to be in the form we stated. If $\overline{\bar{E}}$ is the whole group $\overline{\bar{A}}$, this implies that the inclusion

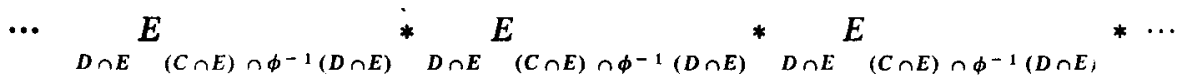

$$
\begin{aligned}
& \subset \cdots * \underset{D C}{A} *{ }_{D}^{A} * \underset{D}{A} C^{*} \cdots
\end{aligned}
$$

is onto, hence $D \cap E=D$ and $C \cap E=C$; and lemma 5 implies that $E=A$. Similarly, we can show that $\vec{F}$ is not the whole group $\overline{\bar{A}}$.

REMARK. This theorem has a natural extension for boundary links with arbitrary components. 


\section{References}

[1] E. M. Brown and R. H. Crowell, 'Deformation retract of 3-manifolds into their boundaries', Ann. of Math. 82 (1965), 445-448.

[2] L. P. Neuwirth, Knot Groups Annals of Mathematical Studies, No. 56 (Princeton University Press) (1965).

[3] C. D. Papakyriakopoulos, 'On solid tori', Proc. London Math. Soc. (3) 1 (1957), 281-299.

[4] C. D. Papakyriakopoulos, 'On Dehn's lemma and the asphericity of knots', Proc. Nat. Acad. Soc. U.S.A. 43 (1957), 169-172.

University of Western Australia

Nedlands, West Australia 6009 